\title{
A new concept of transonic galactic outflows and its application to the Sombrero galaxy
}

\author{
Asuka Igarashi ${ }^{1}$, Masao Mori ${ }^{1,2}$ and Shin-ya Nitta ${ }^{3,4,5}$ \\ ${ }^{1}$ Faculty of Pure and Applied Sciences, University of Tsukuba, \\ 1-1-1, Tennodai, Tsukuba, Ibaraki, 305-8577, Japan \\ e-mail: igarashi@ccs.tsukuba.ac.jp \\ ${ }^{2}$ Center for Computational Sciences, University of Tsukuba, \\ 1-1-1, Tennodai, Tsukuba, Ibaraki, 305-8577, Japan \\ ${ }^{3}$ Tsukuba University of Technology, 4-3-15, Amakubo, Tsukuba, Ibaraki, 305-8520, Japan \\ ${ }^{4}$ Hinode Science Project, National Astronomical Observatory of Japan, \\ 2-21-1 Osawa, Mitaka, Tokyo, 181-8588, Japan \\ ${ }^{5}$ Institute of Space and Astronautical Science, Japan Aerospace Exploration Agency, \\ 3-1-1 Yoshinodai, Sagamihara, \\ Kanagawa, 229-8510, Japan
}

\begin{abstract}
We study fundamental properties of transonic galactic outflows in the gravitational potential of a cold dark matter halo (DMH) with a central super-massive black hole (SMBH) assuming a polytropic, steady and spherically symmetric state. We have classified the transonic solutions with respect to their topology in the phase space. As a result, we have found two types of transonic solutions characterized by a magnitude relationship between the gravity of DMH and that of SMBH. These two types of solutions have different loci of the transonic points; one transonic point is formed at a central region $(<0.01 \mathrm{kpc})$ and another is at a distant region $(>100 \mathrm{kpc})$. Also, mass fluxes and outflow velocities are different between the two solutions. These two transonic solutions may play different roles on the star formation history of galaxies and the metal contamination of intergalactic space. Furthermore, we have applied our model to the Sombrero galaxy. In this galaxy, the wide-spread hot gas is detected as an apparent trace of galactic outflows while the star-formation rate is disproportionately low, and the observed gas density distribution is quite similar to the hydrostatic state ( $\mathrm{Li}$ et al. 2011). To solve this discrepancy, we propose a slowly accelerating outflow in which the transonic point forms in a distant region $(\sim 120 \mathrm{kpc})$ and the subsonic region spreads across the stellar distribution. In the subsonic region, the gas density distribution is similar to that of the hydrostatic state. Our model predicts the possibility of the slowly accelerating outflow in the Sombrero galaxy. Igarashi et al. 2014 used the isothermal model and well reproduced the observed gas density distribution, but the estimated mass flux $\left(1.8 M_{\odot} / \mathrm{yr}\right)$ is lager than the mass of the gas supplied by stars $(0.3-$ $\left.0.4 M_{\odot} / \mathrm{yr}\right)$. Then, we expect that the polytropic model may reproduce the observational mass of the supplied gas (Igarashi et al. 2015). Such slowly accelerating outflows should be distinguished from the conventional supersonic outflows frequently argued in star-forming galaxies.
\end{abstract}

Keywords. hydrodynamics, galaxies:evolution, ISM:jets and outflows, galaxies:ISM, ISM:evolution, galaxies:starburst, galaxies:abundance, galaxies:intergalactic medium, galaxies:individual(NGC4594)

\section{References}

Li, Z., Jones C., Froman, W. R., Kraft R. P., Lal, D. V., Stefano, R. D., Spitler L. R., Tang S.,

Wang Q. D., Gilfanov, M., \& Revnivtsev, M. 2011, ApJ, 730, 84

Igarashi, A., Mori, M., \& Nitta, S. 2014, MNRAS, 444, 1177 